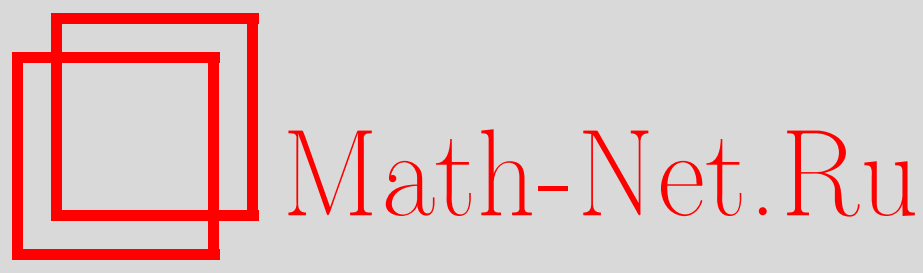

Ю. П. Чубурин, Рассеяние электрона на доменной стенке, ТМФ, 2011, том 166, номер 2, 272-281

DOI: https://doi.org/10.4213/tmf6609

Использование Общероссийского математического портала Math-Net.Ru подразумевает, что вы прочитали и согласны с пользовательским соглашением http://www . mathnet.ru/rus/agreement

Параметры загрузки:

IP: 54.84 .234 .179

26 апреля 2023 г., 16:32:06

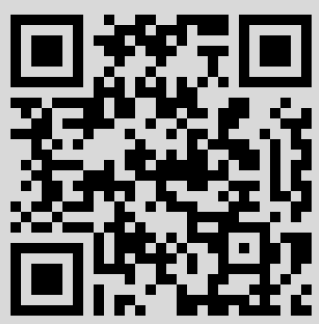




\section{РАССЕЯНИЕ ЭЛЕКТРОНА НА ДОМЕННОЙ СТЕНКЕ}

Для гамильтониана спин-поляризованного электрона, проходящего через доменную стенку в ферромагнитной квантовой проволоке, изучаются коэффициенты прохождения и отражения. Доказано, что для энергий $\lambda \in(-\alpha, \alpha)(-\alpha-$ граница существенного спектра) как для достаточно малых, так и для достаточно больших $\lambda$ имеется почти полное отражение, что согласуется с эффектом баллистического магнитосопротивления в ферромагнитных наноконтактах. Для энергий $\lambda>\alpha$ почти полное отражение сменяется почти полным прохождением; при этом то и другое происходит без переворачивания спина.

Ключевые слова: оператор Шредингера, спин-поляризованный электрон, коэффициенты прохождения и отражения, доменная стенка.

\section{1. ВВЕДЕНИЕ}

В настоящей статье рассматривается оператор Шредингера в $\left(L^{2}(\mathbb{R})\right)^{2}$ вида

$$
H=H_{0}+\alpha V(x),
$$

где

$$
H_{0}=-\frac{d^{2}}{d x^{2}}+\left(\begin{array}{cc}
\alpha \operatorname{sgn} x & 0 \\
0 & -\alpha \operatorname{sgn} x
\end{array}\right), \quad V(x)=\left(\begin{array}{cc}
w(x) & -v(x) \\
-v(x) & -w(x)
\end{array}\right) .
$$

Это математически удобная форма записи гамильтониана

$$
H=-\frac{d^{2}}{d x^{2}}-\alpha \mathbf{M}(x) \cdot \boldsymbol{\sigma}
$$

где $\alpha \neq 0$ - обменный параметр (без ограничения общности считаем, что $\alpha>0$ ), $\mathbf{M}(x)=(v(x), 0,-\operatorname{sgn} x-w(x))$ - вектор намагниченности, $\boldsymbol{\sigma}=\left(\sigma_{1}, \sigma_{2}, \sigma_{3}\right)$ - набор матриц Паули. Здесь $v(x), w(x)$ - ненулевые вещественные функции такие, что

$$
v^{2}(x)+(\operatorname{sgn} x+w(x))^{2}=1
$$

для всех $x \in \mathbb{R}$ и $v(x)=w(x)=0$ для $x \notin[-d, d]$, где $d>0$. Предполагается, что $v(x)$ - четная неотрицательная функция, $w(x)$ - нечетная функция, монотонно

* Физико-технический институт УрО РАН, Ижевск, Россия.

E-mail: chuburin@otf.pti.udm.ru 
возрастающая и неотрицательная на промежутке $[-d, 0)$ и такая, что $|w(x)| \leqslant 1$ (функция $w(x)$ "сглаживает" функцию $\operatorname{sgn} x$ ).

Оператор (1) является гамильтонианом спин-поляризованного электрона, проходящего через доменную стенку в ферромагнитной квантовой проволоке (см., например, [1], [2]). Интерес к таким объектам возник в связи с их возможным применением в наноразмерных магнитоэлектронных устройствах, что связано с экспериментально наблюдаемым эффектом баллистического магнитосопротивления (БМС) (см. работы [3], [4]). Этот эффект состоит в резком увеличении (например, более чем в 30 раз [4]) сопротивления в ферромагнитных наноконтактах при появлении очень малого магнитного поля.

Целью настоящей работы является изучение коэффициентов прохождения и отражения для каждой из спиновых компонент волновой функции. Доказано, что для энергий $\lambda \in(-\alpha, \alpha)$, где $-\alpha$ - граница существенного спектра, как для достаточно малых, так и для достаточно больших $\lambda$ имеется почти полное отражение. Для энергий $\lambda>\alpha$ почти полное отражение сменяется почти полным прохождением, при этом то и другое происходит без переворачивания спина.

Заметим, что почти полное отражение для малых $\alpha$ в промежутке $(-\alpha, \alpha)$ согласуется с эффектом БМС.

\section{2. УРАВНЕНИЕ ЛИППМАНА-ШВИНГЕРА}

Обозначим через $G_{0}(x, y, \lambda, \alpha)$ ядро резольвенты $\left(h_{0}-\lambda\right)^{-1}$ (функцию Грина) оператора $h_{0}=-d^{2} / d x^{2}+\alpha \theta(x)$, где $\theta(x)-$ функция Хевисайда. Очевидно, что функция Грина оператора $H_{0}$ имеет вид

$$
\mathcal{G}_{0}(x, y, \lambda, \alpha)=\left(\begin{array}{cc}
G_{0}(x, y, \lambda+\alpha, 2 \alpha) & 0 \\
0 & G_{0}(x, y, \lambda-\alpha,-2 \alpha)
\end{array}\right) .
$$

Введем обозначения $k=\sqrt{\lambda-\alpha}, k^{\prime}=\sqrt{\lambda+\alpha}$ и положим

$$
G_{01}\left(x, y, k, k^{\prime}\right)=G_{0}(x, y, \lambda+\alpha, 2 \alpha), \quad G_{02}\left(x, y, k, k^{\prime}\right)=G_{0}(x, y, \lambda-\alpha,-2 \alpha) .
$$

В дальнейшем будут использоваться следующие равенства (см. работу [5]):

$$
\begin{aligned}
G_{01}\left(x, y, k, k^{\prime}\right)=-\theta(x) \theta(y)\left(\frac{e^{i k|x-y|}}{2 i k}+\frac{\left(-k^{\prime}+k\right) e^{i k(x+y)}}{2 i k\left(k+k^{\prime}\right)}\right)- \\
\quad-\theta(x) \theta(-y) \frac{e^{i k x-i k^{\prime} y}}{i\left(k+k^{\prime}\right)}-\theta(-x) \theta(y) \frac{e^{-i k^{\prime} x+i k y}}{i\left(k+k^{\prime}\right)}- \\
-\theta(-x) \theta(-y)\left(\frac{e^{i k^{\prime}|x-y|}}{2 i k^{\prime}}-\frac{\left(-k^{\prime}+k\right) e^{-i k^{\prime}(x+y)}}{2 i k^{\prime}\left(k+k^{\prime}\right)}\right)= \\
=-\frac{\left(\theta(x) e^{i k x}+\theta(-x) e^{-i k^{\prime} x}\right)\left(\theta(y) e^{i k y}+\theta(-y) e^{-i k^{\prime} y}\right)}{i\left(k+k^{\prime}\right)}+\gamma_{1}\left(x, y, k, k^{\prime}\right),
\end{aligned}
$$

где

$$
\begin{aligned}
\gamma_{1}\left(x, y, k, k^{\prime}\right)= & -\frac{\theta(x) \theta(y)\left(e^{i k|x-y|}-e^{i k(x+y)}\right)}{2 i k}- \\
& -\frac{\theta(-x) \theta(-y)\left(e^{i k^{\prime}|x-y|}-e^{-i k^{\prime}(x+y)}\right)}{2 i k^{\prime}} .
\end{aligned}
$$


Обозначим через $\chi_{I}(x)$ характеристическую функцию промежутка $I=[-d, d]$. Очевидно, что функция $\sqrt{|w(x)|} \gamma_{1}\left(x, y, k, k^{\prime}\right) \chi_{I}(y)$ является $L^{2}(\mathbb{R})$-значной аналитической функцией переменных $k, k^{\prime}$ в достаточно малой комплексной окрестности нуля в пространстве $\mathbb{C}^{2}$.

Аналогично вводится функция $\gamma_{2}\left(x, y, k, k^{\prime}\right)$. Заметим, что

$$
G_{02}\left(x, y, k, k^{\prime}\right)=G_{01}\left(x, y, k^{\prime}, k\right), \quad \gamma_{2}\left(x, y, k, k^{\prime}\right)=\gamma_{1}\left(x, y, k^{\prime}, k\right) .
$$

Обозначим через $\sigma(A)$ и $\sigma_{\mathrm{ess}}(A)$ спектр и существенный спектр оператора $A$ соответственно.

Доказательство следующей теоремы стандартно (см. доказательство предложения 1 в работе [5]).

Теорема 1. Справедливы следующие равенства:

$$
\sigma_{\mathrm{ess}}(H)=\sigma\left(H_{0}\right)=[-\alpha, \infty) .
$$

Запишем уравнение Липпмана-Швингера в виде

$$
\psi(x)=\psi_{0}(x)-\alpha \int_{\mathbb{R}} \mathcal{G}_{0}(x, y, \lambda+i 0, \alpha) V(y) \psi(y) d y
$$

$(+i 0$ в дальнейшем опускаем), где

$$
\psi(x)=\left(\begin{array}{l}
\psi_{1}(x) \\
\psi_{2}(x)
\end{array}\right), \quad \psi_{0}(x)=\left(\begin{array}{c}
\theta(-x)\left(e^{i k^{\prime} x}+\frac{k^{\prime}-k}{k^{\prime}+k} e^{-i k^{\prime} x}\right)+\theta(x) \frac{2 k^{\prime}}{k+k^{\prime}} e^{i k x} \\
0
\end{array}\right) .
$$

(Функция $\psi_{0}$ удовлетворяет уравнению $\left(H_{0}-\lambda\right) \psi_{0}=0$ и имеет требуемое для описания рассеяния поведение при $x \rightarrow \pm \infty$. Заметим, что выбор

$$
\hat{\psi}_{0}(x)=\left(\theta(-x)\left(e^{i k x}+\frac{k-k^{\prime}}{k^{\prime}+k} e^{-i k x}\right)+\theta(x) \frac{2 k}{k+k^{\prime}} e^{i k^{\prime} x}\right)
$$

малоинтересен, поскольку для $\lambda \in(-\alpha, \alpha)$ волновой вектор $k$ чисто мнимый.)

Обозначим через $a_{1}^{+}\left(k, k^{\prime}\right)$ и $a_{2}^{+}\left(k, k^{\prime}\right)\left(a_{1}^{-}\left(k, k^{\prime}\right)\right.$ и $\left.a_{2}^{-}\left(k, k^{\prime}\right)\right)$ коэффициенты прохождения (отражения) с сохранением и переворачиванием спина соответственно. Из уравнения (5) с учетом формул (4), (6) эти коэффициенты легко находятся. Имеем

$$
\begin{aligned}
a_{1}^{-}\left(k, k^{\prime}\right)= & \frac{k^{\prime}-k}{k^{\prime}+k}+\alpha \int_{0}^{\infty} \frac{e^{i k y}}{i\left(k+k^{\prime}\right)}\left(w(y) \psi_{1}(y)-v(y) \psi_{2}(y)\right) d y+ \\
& +\alpha \int_{-\infty}^{0}\left(\frac{e^{i k^{\prime} y}}{2 i k^{\prime}}-\frac{\left(-k^{\prime}+k\right) e^{-i k^{\prime} y}}{2 i k^{\prime}\left(k+k^{\prime}\right)}\right)\left(w(y) \psi_{1}(y)-v(y) \psi_{2}(y)\right) d y
\end{aligned}
$$

(этот коэффициент отвечает $e^{-i k^{\prime} x}$ ). Для $\lambda>\alpha$

$$
\begin{aligned}
a_{2}^{-}\left(k, k^{\prime}\right)=\alpha & \int_{0}^{\infty} \frac{e^{i k^{\prime} y}}{i\left(k+k^{\prime}\right)}\left(-v(y) \psi_{1}(y)-w(y) \psi_{2}(y)\right) d y+ \\
& +\alpha \int_{-\infty}^{0}\left(\frac{e^{i k y}}{2 i k}-\frac{\left(-k+k^{\prime}\right) e^{-i k y}}{2 i k\left(k+k^{\prime}\right)}\right)\left(-v(y) \psi_{1}(y)-w(y) \psi_{2}(y)\right) d y
\end{aligned}
$$


(этот коэффициент отвечает $e^{-i k x}$; для $\lambda \in(-\alpha, \alpha)$ имеем $a_{2}^{-}\left(k, k^{\prime}\right)=0$, поскольку $e^{-i k x}$ в этом случае экспоненциально убывает). Далее, для $\lambda>\alpha$

$$
\begin{aligned}
a_{1}^{+}\left(k, k^{\prime}\right)= & \frac{2 k^{\prime}}{k^{\prime}+k}+ \\
& +\alpha \int_{0}^{\infty}\left(\frac{e^{-i k y}}{2 i k}+\frac{\left(-k^{\prime}+k\right) e^{i k y}}{2 i k\left(k+k^{\prime}\right)}\right)\left(w(y) \psi_{1}(y)-v(y) \psi_{2}(y)\right) d y+ \\
& +\alpha \int_{-\infty}^{0} \frac{e^{-i k^{\prime} y}}{2 i k^{\prime}}\left(w(y) \psi_{1}(y)-v(y) \psi_{2}(y)\right) d y
\end{aligned}
$$

(этот коэффициент отвечает $e^{i k x} ;$ для $\lambda \in(-\alpha, \alpha)$ имеем $\left.a_{1}^{+}\left(k, k^{\prime}\right)=0\right)$,

$$
\begin{aligned}
a_{2}^{+}\left(k, k^{\prime}\right)= & \alpha \int_{0}^{\infty}\left(\frac{e^{-i k^{\prime} y}}{2 i k^{\prime}}+\frac{\left(-k+k^{\prime}\right) e^{i k^{\prime} y}}{2 i k^{\prime}\left(k+k^{\prime}\right)}\right)\left(-v(y) \psi_{1}(y)-w(y) \psi_{2}(y)\right) d y+ \\
& +\alpha \int_{-\infty}^{0} \frac{e^{-i k y}}{i\left(k+k^{\prime}\right)}\left(-v(y) \psi_{1}(y)-w(y) \psi_{2}(y)\right) d y
\end{aligned}
$$

(этот коэффициент отвечает $e^{i k^{\prime} x}$ ).

Введем обозначения

$$
\begin{gathered}
I_{v}^{ \pm}(\kappa)=\int_{-\infty}^{\infty} \theta( \pm y) v(y) e^{i \kappa y} d y, \quad I_{w}^{ \pm}(\kappa)=\int_{-\infty}^{\infty} \theta( \pm y) v(y) e^{i \kappa y} d y, \\
a\left(k, k^{\prime}\right)=I_{w}^{+}(2 k)+I_{w}^{-}\left(-2 k^{\prime}\right), \\
b\left(k, k^{\prime}\right)=c\left(k, k^{\prime}\right)=I_{v}^{+}\left(k+k^{\prime}\right)+I_{v}^{-}\left(-k-k^{\prime}\right), \\
d\left(k, k^{\prime}\right)=I_{w}^{+}\left(2 k^{\prime}\right)+I_{w}^{-}(-2 k), \\
e\left(k, k^{\prime}\right)=2 k^{\prime} I_{w}^{+}(2 k)+\left(k+k^{\prime}\right) I_{w}^{-}(0)+\left(k^{\prime}-k\right) I_{w}^{-}\left(-2 k^{\prime}\right), \\
f\left(k, k^{\prime}\right)=-2 k^{\prime} I_{v}^{+}\left(k+k^{\prime}\right)-\left(k+k^{\prime}\right) I_{v}^{-}\left(k^{\prime}-k\right)+\left(k-k^{\prime}\right) I_{v}^{-}\left(-k-k^{\prime}\right) .
\end{gathered}
$$

В следующей теореме ищется решение уравнения Липпмана-Швингера в классе функций $\psi$ таких, что $\sqrt{|w|} \psi \in\left(L^{2}(\mathbb{R})\right)^{2}$.

Теорема 2. Пусть $\lambda>0$ фиксировано (и не зависит от $\alpha$ ) или $\lambda \in(-\alpha, \alpha)$. Тогда для всех достаточно малых а существует единственное решение уравнения Липпмана-Швингера $(5) \psi \in\left(L^{\infty}(\mathbb{R})\right)^{2}$ вида

$$
\psi(x)=\psi_{0}(x)+\left(\begin{array}{l}
C_{1}\left(\theta(x) e^{i k x}+\theta(-x) e^{-i k^{\prime} x}\right) \\
C_{2}\left(\theta(x) e^{i k^{\prime} x}+\theta(-x) e^{-i k x}\right)
\end{array}\right)+O(\alpha),
$$

где константы $C_{1}, C_{2}$ находятся из системы

$$
\begin{aligned}
& \left(\begin{array}{cc}
i\left(k+k^{\prime}\right)-\alpha a\left(k, k^{\prime}\right)+O\left(\alpha^{2}\right) & \alpha b\left(k, k^{\prime}\right)+O\left(\alpha^{2}\right) \\
\alpha c\left(k, k^{\prime}\right)+O\left(\alpha^{2}\right) & i\left(k+k^{\prime}\right)+\alpha d\left(k, k^{\prime}\right)+O\left(\alpha^{2}\right)
\end{array}\right)\left(\begin{array}{l}
C_{1} \\
C_{2}
\end{array}\right)= \\
& \quad=\frac{\alpha}{k+k^{\prime}}\left(\begin{array}{c}
e\left(k, k^{\prime}\right)+O(\alpha) \\
f\left(k, k^{\prime}\right)+O(\alpha)
\end{array}\right),
\end{aligned}
$$

а $O(\alpha)$ имеет следующий смысл:

$$
\|\sqrt{|w|} O(\alpha)\|_{\left(L^{2}(\mathbb{R})\right)^{2}} \leqslant C \alpha .
$$


ДоказАтЕльСтво. Из соотношения (2) имеем $v(x)=\sqrt{|w(x)|} \sqrt{2-|w(x)|}$. Введем обозначения (ср. с книгой $[6])$

$$
\begin{aligned}
& \sqrt{w(x)}=\sqrt{|w(x)|} \operatorname{sgn} w(x)=-\sqrt{|w(x)|} \operatorname{sgn} x, \\
& \varphi_{j}=\sqrt{|w|} \psi_{j}, \quad j=1,2, \quad \varphi_{01}=\sqrt{|w|} \psi_{01} .
\end{aligned}
$$

С учетом формул (4), (6) уравнение (5) для новой неизвестной функции $\varphi=\left(\varphi_{1}, \varphi_{2}\right)^{\mathrm{T}}$ примет вид

$$
\begin{aligned}
& \left(\begin{array}{l}
\varphi_{1}(x) \\
\varphi_{2}(x)
\end{array}\right)=\left(\begin{array}{c}
\varphi_{01}(x) \\
0
\end{array}\right)+\frac{\alpha \sqrt{|w(x)|}}{i\left(k+k^{\prime}\right)} \times \\
& \quad \times \int_{\mathbb{R}}\left(\theta(x)\left(\begin{array}{cc}
e^{i k x}\left(\theta(y) e^{i k y}+\theta(-y) e^{-i k^{\prime} y}\right) \\
0 & e^{i k^{\prime} x}\left(\theta(y) e^{i k^{\prime} y}+\theta(-y) e^{-i k y}\right)
\end{array}\right)+\right. \\
& \quad+\theta(-x)\left(\begin{array}{cc}
e^{-i k^{\prime} x}\left(\theta(y) e^{i k y}+\theta(-y) e^{-i k^{\prime} y}\right) \\
0
\end{array}\right) \\
& \quad \times\left(\begin{array}{c}
\sqrt{w(y)} \varphi_{1}(y)-\sqrt{2-|w(y)|} \varphi_{2}(y) \\
-\sqrt{2-|w(y)|} \varphi_{1}(y)-\sqrt{w(y)} \varphi_{2}(y)
\end{array}\right) d y+\alpha K\left(k, k^{\prime}\right) W\left(\begin{array}{c}
\varphi_{1}(x) \\
\varphi_{2}(x)
\end{array}\right)
\end{aligned}
$$

где $K\left(k, k^{\prime}\right)$ - оператор Гильберта-Шмидта с ядром

$$
\begin{gathered}
\left(\begin{array}{cc}
\sqrt{|w(x)|} \gamma_{1}\left(x, y, k, k^{\prime}\right) \chi_{I}(y) & 0 \\
0 & \sqrt{|w(x)|} \gamma_{2}\left(x, y, k, k^{\prime}\right) \chi_{I}(y)
\end{array}\right), \\
W=\left(\begin{array}{cc}
\sqrt{w(x)} & -\sqrt{2-|w(x)|} \chi_{I}(x) \\
-\sqrt{2-|w(x)|} \chi_{I}(x) & -\sqrt{w(x)}
\end{array}\right) .
\end{gathered}
$$

Положим по определению

$$
\xi=\left(\begin{array}{l}
\xi_{1} \\
\xi_{2}
\end{array}\right)=\left(1-\alpha K\left(k, k^{\prime}\right) W\right) \varphi .
$$

Для малых $\alpha$ перепишем уравнение (14) в виде

$$
\begin{aligned}
& \xi(x)=\varphi_{0}(x)+\frac{\alpha \sqrt{|w(x)|}}{i\left(k+k^{\prime}\right)} \times \\
& \times \int_{\mathbb{R}}\left(\theta(x)\left(\begin{array}{cc}
e^{i k x}\left(\theta(y) e^{i k y}+\theta(-y) e^{-i k^{\prime} y}\right) & 0 \\
0 & e^{i k^{\prime} x}\left(\theta(y) e^{i k^{\prime} y}+\theta(-y) e^{-i k y}\right)
\end{array}\right)+\right. \\
& \left.+\theta(-x)\left(\begin{array}{cc}
e^{-i k^{\prime} x}\left(\theta(y) e^{i k y}+\theta(-y) e^{-i k^{\prime} y}\right) & 0 \\
0 & e^{-i k x}\left(\theta(y) e^{i k^{\prime} y}+\theta(-y) e^{-i k y}\right)
\end{array}\right)\right) \times \\
& \times W\left(1-\alpha K\left(k, k^{\prime}\right) W\right)^{-1} \xi(y) d y .
\end{aligned}
$$

Следовательно,

$$
\xi(x)=\varphi_{0}(x)+\sqrt{|w(x)|}\left(\begin{array}{l}
C_{1}\left(\theta(x) e^{i k x}+\theta(-x) e^{-i k^{\prime} x}\right) \\
C_{2}\left(\theta(x) e^{i k^{\prime} x}+\theta(-x) e^{-i k x}\right)
\end{array}\right),
$$


где $C_{1}, C_{2}$ - некоторые константы. Из выражений $(16),(17)$ получаем систему

$$
\begin{aligned}
C_{1}= & \frac{\alpha}{i\left(k+k^{\prime}\right)} \int_{\mathbb{R}}\left(\theta(y) e^{i k y}+\theta(-y) e^{-i k^{\prime} y}\right) \times \\
& \times\left[w(y)\left(\theta(y) e^{i k y}+\theta(-y) e^{-i k^{\prime} y}\right) C_{1}-v(y)\left(\theta(y) e^{i k^{\prime} y}+\theta(-y) e^{-i k y}\right) C_{2}+\right. \\
& +w(y)\left(\theta(-y)\left(e^{i k^{\prime} y}+\frac{k^{\prime}-k}{k^{\prime}+k} e^{-i k^{\prime} y}\right)+\theta(y) \frac{2 k^{\prime}}{k^{\prime}+k} e^{i k y}\right)+ \\
& \left.+\alpha\left(a_{11}(y, \alpha) C_{1}+a_{12}(y, \alpha) C_{2}+b_{1}(y, \alpha)\right)\right] d y \\
C_{2}= & \frac{\alpha}{i\left(k+k^{\prime}\right)} \int_{\mathbb{R}}\left(\theta(y) e^{i k^{\prime} y}+\theta(-y) e^{-i k y}\right) \times \\
& \times\left[-v(y)\left(\theta(y) e^{i k y}+\theta(-y) e^{-i k^{\prime} y}\right) C_{1}-w(y)\left(\theta(y) e^{i k^{\prime} y}+\theta(-y) e^{-i k y}\right) C_{2}-\right. \\
& -v(y)\left(\theta(-y)\left(e^{i k^{\prime} y}+\frac{k^{\prime}-k}{k^{\prime}+k} e^{-i k^{\prime} y}\right)+\theta(y) \frac{2 k^{\prime}}{k^{\prime}+k} e^{i k y}\right)+ \\
& \left.+\alpha\left(a_{21}(y, \alpha) C_{1}+a_{22}(y, \alpha) C_{2}+b_{2}(y, \alpha)\right)\right] d y,
\end{aligned}
$$

где $a_{i j}(y, \alpha), b_{i}(y, \alpha)$ - равномерно ограниченные по $\alpha$ семейства функций с носителями в $I$. Легко видеть, что систему (18) можно записать в виде (12). Используя выражение (15) и (17), получаем формулу (11) и неравенство (13). Ограниченность функции $\psi$ легко вытекает из уравнения (5) с учетом формул (4), (6).

Для завершения доказательства покажем, что определитель системы (12) не равен нулю для достаточно малых $\alpha$. Если $\lambda>0$ фиксировано, то, очевидно, определитель стремится к $-4 \lambda \neq 0$ при $\alpha \rightarrow 0$. Предположим теперь, что $-\alpha<\lambda<\alpha$, тогда $k=O(\sqrt{\alpha}), k^{\prime}=O(\sqrt{\alpha})$. Заметим, что

$$
\begin{gathered}
I_{w}^{+}(2 k)+I_{w}^{-}\left(-2 k^{\prime}\right)=I_{w}^{+}(0)+I_{w}^{-}\left(0^{\prime}\right)+O(\sqrt{\alpha})= \\
=\int_{\mathbb{R}} w(x) d x+O(\sqrt{\alpha})=O(\sqrt{\alpha}), \\
I_{w}^{+}\left(2 k^{\prime}\right)+I_{w}^{-}(-2 k)=O(\sqrt{\alpha}), \quad I_{v}^{+}\left(k+k^{\prime}\right)+I_{v}^{-}\left(-k-k^{\prime}\right)=\bar{v}+O(\sqrt{\alpha}),
\end{gathered}
$$

где $\bar{v}=\int_{\mathbb{R}} v(x) d x$. Далее, в силу сделанного предположения $\left|k+k^{\prime}\right|^{2}=\mid i \sqrt{\alpha-\lambda}+$ $\left.\sqrt{\lambda+\alpha}\right|^{2}=2 \alpha$. Следовательно, определитель системы (12)

$$
\left|\begin{array}{cc}
i\left(k+k^{\prime}\right)+O\left(\alpha^{3 / 2}\right) & \alpha \bar{v}+O\left(\alpha^{3 / 2}\right) \\
\alpha \bar{v}+O\left(\alpha^{3 / 2}\right) & i\left(k+k^{\prime}\right)+O\left(\alpha^{3 / 2}\right)
\end{array}\right|=-\left(k+k^{\prime}\right)^{2}+O\left(\alpha^{2}\right)=2 \alpha e^{i \varphi}+O\left(\alpha^{2}\right) \neq 0
$$

для малых $\alpha$ (здесь $\varphi \in \mathbb{R})$. Теорема доказана.

СЛЕДСТвИЕ. В случае фиксированного $\lambda>0$ имеем $\psi=\psi_{0}+O(\alpha)$.

ЗАмЕчание 1 . Обозначим через $R_{0}(\lambda)$ и $R(\lambda)$ резольвенты операторов $H_{0}$ и $H$ соответственно. Нетрудно видеть (ср. с работой [5]), что операторы $\sqrt{V} R_{0}(\lambda)$, $R_{0}(\lambda) \sqrt{V}$, а значит, и оператор

$$
\sqrt{V} R(i)=\sqrt{V} R_{0}(i)-\sqrt{V} R_{0}(i) V R(i)
$$


являются операторами Гильберта-Шмидта. Но тогда оператор

$$
R(i)-R_{0}(i)=-R_{0}(i) V R(i)
$$

является оператором со следом. Из теоремы Куроды-Бирмана [6] вытекает, что волновые операторы $\Omega^{ \pm}\left(H, H_{0}\right)$ существуют и полны, а следовательно, существует унитарный оператор рассеяния.

\section{3. РАССЕЯНИЕ ДЛЯ МАЛЫХ $\alpha$}

Обозначим через $P_{1}^{+}\left(k, k^{\prime}\right)=\left|a_{1}^{+}\left(k, k^{\prime}\right)\right|^{2}$ и $P_{2}^{+}\left(k, k^{\prime}\right)=\left|a_{2}^{+}\left(k, k^{\prime}\right)\right|^{2}\left(P_{1}^{-}\left(k, k^{\prime}\right)=\right.$ $\left|a_{1}^{-}\left(k, k^{\prime}\right)\right|^{2}$ и $\left.P_{2}^{-}\left(k, k^{\prime}\right)=\left|a_{2}^{-}\left(k, k^{\prime}\right)\right|^{2}\right)$ вероятности прохождения (отражения) с сохранением и переворачиванием спина соответственно.

Теорема 3. Пусть $\lambda>0$ фиксировано. Тогда

$$
P_{1}^{+}\left(k, k^{\prime}\right)=1+O(\alpha), \quad P_{2}^{+}\left(k, k^{\prime}\right)=O\left(\alpha^{2}\right) .
$$

ДокАЗАТЕЛьство. С помощью формул (9), (10), а также следствия теоремы 2 получаем равенства

$$
a_{1}^{+}\left(k, k^{\prime}\right)=\frac{2 k^{\prime}}{k+k^{\prime}}+O(\alpha)=1+O(\alpha), \quad a_{2}^{+}\left(k, k^{\prime}\right)=O(\alpha),
$$

которые и доказывают теорему.

ЗАмечание 2. Легко видеть, что оценка $|O(\alpha)| \leqslant C|\alpha|$ равномерна по $\lambda \in[a, b]$, где $a>0$.

Теорема 4. Предположим, что $\lambda \in(-\alpha, \alpha)$. Тогда

$$
P_{1}^{-}\left(k, k^{\prime}\right)=1+O(\alpha), \quad P_{2}^{-}\left(k, k^{\prime}\right)=0,
$$

причем в оценке $|O(\alpha)| \leqslant C \alpha$ константа $C$ не зависит от $\lambda$.

ДокАЗАТЕЛЬство. В рассматриваемом случае система (12) примет вид (см. доказательство теоремы 2)

$$
\begin{aligned}
& \left(\begin{array}{cc}
i\left(k+k^{\prime}\right)+O\left(\alpha^{3 / 2}\right) & \alpha \bar{v}+O\left(\alpha^{3 / 2}\right) \\
\alpha \bar{v}+O\left(\alpha^{3 / 2}\right) & i\left(k+k^{\prime}\right)+O\left(\alpha^{3 / 2}\right)
\end{array}\right)\left(\begin{array}{l}
C_{1} \\
C_{2}
\end{array}\right)= \\
& =\frac{\alpha}{k+k^{\prime}}\left(\begin{array}{c}
O(\alpha) \\
-2 k^{\prime} \bar{v}+O(\alpha)
\end{array}\right) .
\end{aligned}
$$

Отсюда, учитывая, что $\left|k+k^{\prime}\right|=\sqrt{2 \alpha}$, находим

$$
\begin{aligned}
& C_{1}=\frac{\alpha O\left(\alpha^{3 / 2}\right)}{\left(-\left(k+k^{\prime}\right)^{2}+O\left(\alpha^{2}\right)\right)\left(k+k^{\prime}\right)}=O(\alpha), \\
& C_{2}=\frac{\alpha O(\alpha)}{\left(-\left(k+k^{\prime}\right)^{2}+O\left(\alpha^{2}\right)\right)\left(k+k^{\prime}\right)}=O\left(\alpha^{1 / 2}\right) .
\end{aligned}
$$

Согласно формулам (6), (11) и (20) получаем

$$
\psi(x)=\left(\begin{array}{c}
\theta(-x)\left(e^{i k^{\prime} x}+\frac{k^{\prime}-k}{k^{\prime}+k} e^{-i k^{\prime} x}\right)+\theta(x) \frac{2 k^{\prime}}{k+k^{\prime}} e^{i k x}+O(\alpha) \\
O\left(\alpha^{1 / 2}\right)
\end{array}\right) .
$$


Используя формулу (7), имеем

$$
\begin{aligned}
a_{1}^{-}\left(k, k^{\prime}\right)= & \frac{k^{\prime}-k}{k^{\prime}+k}+\frac{\alpha}{i\left(k+k^{\prime}\right)} \int_{0}^{\infty} w(y) \frac{2 k^{\prime}}{k^{\prime}+k} d y+ \\
& +\frac{\alpha}{2 i k^{\prime}} \int_{-\infty}^{0} \frac{2 k^{\prime}}{k+k^{\prime}} w(y) \frac{2 k^{\prime}}{k+k^{\prime}} d y+O(\alpha)=\frac{k^{\prime}-k}{k^{\prime}+k}+O(\alpha) .
\end{aligned}
$$

Поскольку

$$
\left|\frac{k^{\prime}-k}{k^{\prime}+k}\right|=\left|\frac{k^{\prime 2}-k^{2}}{\left(k^{\prime}+k\right)^{2}}\right|=1,
$$

из формулы (21) вытекает первое из равенств (19). Второе равенство получаем из равенства $a_{2}^{-}\left(k, k^{\prime}\right)=0$ (см. объяснение после $\left.(7)\right)$. То, что константа $C$ в оценке $O(\alpha)$ не зависит от $\lambda$, следует из оценок величин $k, k^{\prime}, k+k^{\prime}$ (см. конец доказательства теоремы 2) и аналитичности $K\left(k, k^{\prime}\right)$. Теорема доказана.

\section{4. РАССЕЯНИЕ ДЛЯ БОЛЬШИХ $\alpha$}

Рассмотрим теперь случай $\alpha \gg 1$. Предположим, что функции $v(x)$ и $w(x)$ дополнительно обладают следующими свойствами: функция $\alpha v(x)$ близка к $a \delta(x)$ для некоторого $a \in \mathbb{R}$, а функция $\alpha w(x)$ близка к нулю (в смысле теории обобщенных функций). Покажем, что такие функции существуют. Выберем последовательности положительных чисел $\alpha_{n} \rightarrow \infty$ и $d_{n} \rightarrow 0(n \rightarrow \infty)$, а также функции $v(x)=v_{n}(x)$, $n=1,2, \ldots$, удовлетворяющие, помимо ранее наложенных условий, оценкам

$$
\frac{a}{2}=\alpha_{n} \int_{0}^{d_{n}} v_{n}(x) d x \geqslant C_{0} \alpha_{n} d_{n},
$$

где $a, C_{0}>0$ - некоторые константы, не зависящие от $n$. Тогда

$$
\lim _{n \rightarrow \infty} \alpha_{n} v_{n}=a \delta(x)
$$

в смысле сходимости обобщенных функций. Далее, для соответствующих функций $w_{n}(x)$ имеем

$$
\alpha_{n} \int_{0}^{d_{n}}\left|w_{n}(x)\right| d x \leqslant \alpha_{n} d_{n} \leqslant \frac{a}{2 C_{0}} .
$$

Поэтому

$$
\begin{aligned}
\alpha_{n} w_{n}(x) & =\theta(-x) \alpha_{n} w_{n}(x)+\theta(x) \alpha_{n} w_{n}(x)= \\
& =\alpha_{n} \int_{0}^{d_{n}}\left|w_{n}(y)\right| d y\left(\frac{\theta(-x) \alpha_{n} w_{n}(x)}{\alpha_{n} \int_{0}^{d_{n}}\left|w_{n}(y)\right| d y}+\frac{\theta(x) \alpha_{n} w_{n}(x)}{\alpha_{n} \int_{0}^{d_{n}}\left|w_{n}(y)\right| d y}\right) \rightarrow 0
\end{aligned}
$$

при $n \rightarrow \infty$ в смысле обобщенных функций.

При сделанных предположениях заменим гамильтониан (1) на оператор вида

$$
H^{\prime}=-\frac{d^{2}}{d x^{2}}+\alpha \operatorname{sgn} x \sigma_{3}-a \delta(x) \sigma_{1} .
$$


Уравнение Липпмана-Швингера (5) примет вид (с учетом непрерывности функции $G_{0}$ в точке $y=0$ )

$$
\begin{aligned}
\left(\begin{array}{c}
\psi_{1}(x) \\
\psi_{2}(x)
\end{array}\right)= & \left(\begin{array}{c}
\theta(-x)\left(e^{i k^{\prime} x}+\frac{k^{\prime}-k}{k^{\prime}+k} e^{-i k^{\prime} x}\right)+\theta(x) \frac{2 k^{\prime}}{k+k^{\prime}} e^{i k x} \\
0
\end{array}\right)+ \\
& +\frac{a \theta(x)}{i\left(k+k^{\prime}\right)}\left(\begin{array}{c}
-e^{i k x} \psi_{2}(0) \\
-e^{i k^{\prime} x} \psi_{1}(0)
\end{array}\right)+\frac{a \theta(-x)}{i\left(k+k^{\prime}\right)}\left(\begin{array}{l}
-e^{-i k^{\prime} x} \psi_{2}(0) \\
-e^{-i k x} \psi_{1}(0)
\end{array}\right) .
\end{aligned}
$$

Отсюда получаем линейную систему относительно $\psi_{1}(0), \psi_{2}(0)$ вида

$$
\left(\begin{array}{cc}
1 & \frac{a}{i\left(k+k^{\prime}\right)} \\
\frac{a}{i\left(k+k^{\prime}\right)} & 1
\end{array}\right)\left(\begin{array}{l}
\psi_{1}(0) \\
\psi_{2}(0)
\end{array}\right)=\left(\begin{array}{c}
\frac{2 k^{\prime}}{k+k^{\prime}} \\
0
\end{array}\right) .
$$

Следовательно,

$$
\psi_{1}(0)=\frac{2 k^{\prime}\left(k+k^{\prime}\right)}{\left(k+k^{\prime}\right)^{2}+a^{2}}, \quad \psi_{2}(0)=\frac{2 i a k^{\prime}}{\left(k+k^{\prime}\right)^{2}+a^{2}} .
$$

Теорема 5. Предположим, что $\lambda=A \alpha^{p}$, где $A>0, p>1$. Тогда для всех достаточно больиих $\alpha$

$$
P_{1}^{+}\left(k, k^{\prime}\right)=1+O\left(\frac{1}{\alpha^{p-1}}\right), \quad P_{2}^{+}\left(k, k^{\prime}\right)=O\left(\frac{1}{\alpha^{p}}\right) .
$$

ДоказАТЕЛьство. Имеем

$$
\begin{aligned}
& k=\sqrt{A \alpha^{p}-\alpha}=\sqrt{A \alpha^{p}}+O\left(\alpha^{1-p / 2}\right), \\
& k^{\prime}=\sqrt{A \alpha^{p}+\alpha}=\sqrt{A \alpha^{p}}+O\left(\alpha^{1-p / 2}\right) .
\end{aligned}
$$

Из (22), (23) находим

$$
\begin{aligned}
a_{1}^{+}\left(k, k^{\prime}\right) & =\frac{2 k^{\prime}}{k+k^{\prime}}-\frac{a}{i\left(k+k^{\prime}\right)} \frac{2 i a k^{\prime}}{\left(k+k^{\prime}\right)^{2}+a^{2}}= \\
& =\frac{2 k^{\prime}\left(k+k^{\prime}\right)}{\left(k+k^{\prime}\right)^{2}+a^{2}}=\frac{4 A \alpha^{p}+O(\alpha)}{4 A \alpha^{p}+O(\alpha)+a^{2}}=1+O\left(\frac{1}{\alpha^{p-1}}\right),
\end{aligned}
$$

что доказывает первое утверждение теоремы, и

$$
\begin{aligned}
a_{2}^{+}\left(k, k^{\prime}\right) & =-\frac{a}{i\left(k+k^{\prime}\right)} \frac{2 k^{\prime}\left(k+k^{\prime}\right)}{\left(k+k^{\prime}\right)^{2}+a^{2}}= \\
& =\frac{2 i a k^{\prime}}{\left(k+k^{\prime}\right)^{2}+a^{2}}=O\left(\frac{1}{\alpha^{p / 2}}\right)
\end{aligned}
$$

что доказывает второе утверждение.

Теорема 6. Предположим, что $\lambda \in(-\alpha, \alpha)$. Тогда для всех достаточно больuux $\alpha$

$$
P_{1}^{-}\left(k, k^{\prime}\right)=1+O\left(\frac{1}{\alpha}\right), \quad P_{2}^{-}\left(k, k^{\prime}\right)=0 .
$$


ДокАЗАтЕЛЬСтво. Вследствие соотношений $(22)$ и $(23)$ имеем $a_{2}^{-}\left(k, k^{\prime}\right)=0$. Для доказательства первого из равенств (24) найдем

$$
a_{1}^{-}\left(k, k^{\prime}\right)=\frac{k^{\prime}-k}{k+k^{\prime}}-\frac{a}{i\left(k+k^{\prime}\right)} \frac{2 i a k^{\prime}}{\left(k+k^{\prime}\right)^{2}+a^{2}} .
$$

Далее,

$$
\left|k+k^{\prime}\right|=\sqrt{2 \alpha}, \quad k^{\prime}=O(\sqrt{\alpha}), \quad\left|\frac{k^{\prime}-k}{k+k^{\prime}}\right|=1,
$$

откуда вытекает первое равенство. Второе равенство является следствием равенства $a_{2}^{-}\left(k, k^{\prime}\right)=0$. Теорема доказана.

\section{Список литературы}

[1] V. K. Dugaev, J. Barnaś, J. Berakdar, J. Phys. A, 36:35 (2003), 9263-9274.

[2] R. Sýkora, I. Turek, Acta Phys. Polonica A, 113:1 (2008), 15-18.

[3] N. García, M. Muñoz, Y.-W. Zhao, Phys. Rev. Lett., 82:14 (1999), 2923-2926.

[4] H. D. Chopra, S. Z. Hua, Phys. Rev. B, 66:2 (2002), 020403(R), 3 pp.

[5] Ю. П. Чубурин, ТМФ, 120:2 (1999), 277-290.

[6] М. Рид, Б. Саймон, Методы современной математической физики, т. 3: Теория рассеяния, Мир, М., 1982.

Поступила в редакцию 8.06.2010, после доработки 30.08.2010 